\title{
Persistent spin currents in mesoscopic Heisenberg rings
}

\author{
Florian Schütz, Marcus Kollar, and Peter Kopietz \\ Institut für Theoretische Physik, Universität Frankfurt, \\ Robert-Mayer-Strasse 8, 60054 Frankfurt, Germany
}

(Dated: July 7, 2003)

\begin{abstract}
We show that at low temperatures $T$ an inhomogeneous radial magnetic field with magnitude $B$ gives rise to a persistent magnetization current around a mesoscopic ferromagnetic Heisenberg ring. Under optimal conditions this spin current can be as large as $g \mu_{\mathrm{B}}(T / \hbar) \exp \left[-2 \pi\left(g \mu_{\mathrm{B}} B / \Delta\right)^{1 / 2}\right]$, as obtained from leading-order spin-wave theory. Here $g$ is the gyromagnetic factor, $\mu_{\mathrm{B}}$ is the Bohr magneton, and $\Delta$ is the energy gap between the ground state and the first spin-wave excitation. The magnetization current endows the ring with an electric dipole moment.
\end{abstract}

PACS numbers: 75.10.Jm, 75.10.Pq, 75.30.Ds, 73.23.Ra

The controlled fabrication of submicron devices has opened the door to a rich new field of theoretical and experimental physics. At low temperatures these devices are mesoscopic in the sense that their quantum states must be described by coherent wave functions extending over the entire system. Then the usual assumptions underlying the averaging procedure in statistical mechanics are not necessarily valid, and quantum-mechanical interference effects become important [1].

A prominent example is persistent currents in mesoscopic normal metal rings threaded by a magnetic flux 1]. Although this phenomenon was predicted long ago 2, 3], the experimental difficulties in measuring persistent currents in an Aharonov-Bohm geometry were only overcome in the past decade [4, 5, 6]. Surprisingly, for metallic rings in the diffusive regime the observed currents were much larger than predicted by theory 1 ] On the other hand, in the ballistic regime [6] the order of magnitude of the observed current can be explained with a simple model of free fermions moving on a ring pierced by a magnetic flux $\phi$. Then the stationary energies are $\epsilon_{n}=\hbar^{2} k_{n}^{2} / 2 m_{*}$, where $k_{n}=\frac{2 \pi}{L}\left(n-\frac{\phi}{\phi_{0}}\right)$, $n=0, \pm 1, \pm 2, \ldots$, are the allowed wavevectors for a ring with circumference $L$. Here $\phi_{0}=h c / e$ is the flux quantum and $m_{*}$ is the effective mass of the electrons. In the simplest approximation, one may calculate the current $I=-c \partial \Omega_{\mathrm{gc}}(\phi) / \partial \phi$ at constant chemical potential $\mu$ from the flux-dependent part of the grand canonical potential $\Omega_{\mathrm{gc}}(\phi)$. At finite temperature $T$, one obtains for spinless fermions [7]

$$
I=\frac{-e}{L} \sum_{n} \frac{v_{n}}{e^{\left(\epsilon_{n}-\mu\right) / T}+1}
$$

where $v_{n}=\hbar k_{n} / m_{*}$. For $T \ll \mu$ the amplitude of the current is $I_{\max } \approx-e v_{F} / L$ (where $v_{F}$ is the Fermi velocity), in agreement with experiment $[\underline{6}$.

In this Letter, we show that Heisenberg spin chains in inhomogeneous magnetic fields can be used to realize a spin current analogue of mesoscopic persistent currents in normal metal rings. Note that in the presence of spin-orbit coupling spin currents in spin chains can also be driven by inhomogeneous electric fields [8], due to the Aharonov-Casher effect [9]. As detailed later on, the magnetization current is carried by magnons and endows the ring with an electric dipole field, which is the counterpart of the magnetic dipole field associated with the persistent charge current in a normal metal ring. We find that for realistic parameters the spin analogues of the experiments in Refs. 4, 5, 6 require the detection of a potential drop on the order of nanovolts.

Due to its relevance for information processing based on spin degrees of freedom, the problem of magnetization transport has received a lot of attention recently [10], especially for systems where spin currents are carried by itinerant electrons 11, 12, 13, 14, 15, 16, 17. Magnetic insulators also show interesting spin transport phenomena [18, 19]. Very recently, Meier and Loss [19] calculated the mesoscopic spin conductance for Heisenberg-type systems in a two-terminal geometry. Here we consider the same problem in a ring geometry and for inhomogeneous magnetic fields that lead to noncoplanar spin configurations. It is known that such configurations can lead to dissipationless transport of charge and spin [1, 14, 15]. In the following, we use linear spin-wave theory to derive the mesoscopic persistent spin current circulating in the ring, corresponding to an infinite spin conductance, and explicitly obtain the associated electric field.

Let us start with a general Heisenberg Hamiltonian

$$
\hat{H}=\frac{1}{2} \sum_{i, j} J_{i j} \boldsymbol{S}_{i} \cdot \boldsymbol{S}_{j}-g \mu_{\mathrm{B}} \sum_{i} \boldsymbol{B}_{i} \cdot \boldsymbol{S}_{i},
$$

where the sums are over all sites $\boldsymbol{r}_{i}$ of a chain with periodic boundary conditions, $J_{i j}$ are general exchange couplings, and $\boldsymbol{S}_{i}$ are spin- $S$ operators normalized such that $\boldsymbol{S}_{i}^{2}=S(S+1)$. The last term in Eq. (2) is the Zeeman energy associated with an inhomogeneous magnetic field $\boldsymbol{B}_{i}=\boldsymbol{B}\left(\boldsymbol{r}_{i}\right)$. We assume that the magnetic field at each lattice site is sufficiently strong to induce permanent magnetic dipole moments $\boldsymbol{m}_{i}=g \mu_{\mathrm{B}}\left\langle\boldsymbol{S}_{i}\right\rangle$, not necessarily parallel to $\boldsymbol{B}_{i}$, where $\langle\ldots\rangle$ denotes the usual thermal average. Moreover, we assume that the unit vectors $\hat{\boldsymbol{m}}_{i}$ $=\boldsymbol{m}_{i} /\left|\boldsymbol{m}_{i}\right|$ trace out a finite solid angle $\Omega$ on the unit 


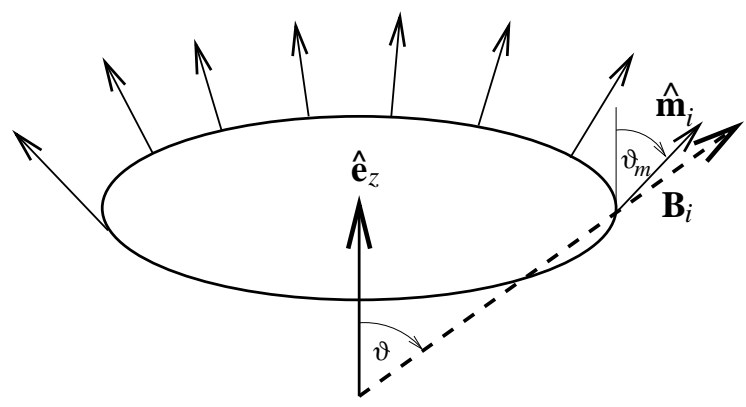

FIG. 1: Classical spin configuration $\hat{\boldsymbol{m}}_{i}$ of a nearest-neighbor ferromagnetic Heisenberg ring in a radial magnetic field $\boldsymbol{B}_{i}$.

sphere in order-parameter space as we move once around the chain. The simplest geometry is a ferromagnetic ring in a crown-shaped magnetic field, as illustrated in Fig. 1

In the general case, the Hamiltonian (2) implies the equation of motion

$$
\hbar \frac{\partial \boldsymbol{S}_{i}}{\partial t}+\boldsymbol{h}_{i} \times \boldsymbol{S}_{i}+\sum_{j} \boldsymbol{I}_{i \rightarrow j}=0
$$

where $\boldsymbol{h}_{i}=g \mu_{\mathrm{B}} \boldsymbol{B}_{i}$, and $\boldsymbol{I}_{i \rightarrow j}=J_{i j} \boldsymbol{S}_{i} \times \boldsymbol{S}_{j}$ is the spin current from site $\boldsymbol{r}_{i}$ to site $\boldsymbol{r}_{j}$. From Eq. (3) it is easy to show that in equilibrium $\sum_{j} \hat{\boldsymbol{m}}_{i} \cdot\left\langle\boldsymbol{I}_{i \rightarrow j}\right\rangle=0$ which is Kirchhoff's law for spin currents [20].

It is convenient to decompose the spin operators as $\boldsymbol{S}_{i}=S_{i}^{\|} \hat{\boldsymbol{m}}_{i}+\boldsymbol{S}_{i}^{\perp}$, with $\boldsymbol{S}_{i}^{\perp} \cdot \hat{\boldsymbol{m}}_{i}=0$. Substituting this into Eq. (2) we obtain $\hat{H}=\hat{H}^{\|}+\hat{H}^{\perp}+\hat{H}^{\prime}$, with

$$
\begin{aligned}
\hat{H}^{\|} & =\frac{1}{2} \sum_{i, j} J_{i j} \hat{\boldsymbol{m}}_{i} \cdot \hat{\boldsymbol{m}}_{j} S_{i}^{\|} S_{j}^{\|}-\sum_{i} \boldsymbol{h}_{i} \cdot \hat{\boldsymbol{m}}_{i} S_{i}^{\|}, \\
\hat{H}^{\perp} & =\frac{1}{2} \sum_{i, j} J_{i j} \boldsymbol{S}_{i}^{\perp} \cdot \boldsymbol{S}_{j}^{\perp} \\
\hat{H}^{\prime} & =-\sum_{i} \boldsymbol{S}_{i}^{\perp} \cdot\left(\boldsymbol{h}_{i}-\sum_{j} J_{i j} S_{j}^{\|} \hat{\boldsymbol{m}}_{j}\right) .
\end{aligned}
$$

To develop the standard semiclassical spin-wave theory, we expand in the small parameter $1 / S$. The leadingorder term [i.e., Eq. (4) with $S_{i}^{\|}$replaced by $S$ ] yields the classical energy. A necessary condition for its minimum is the invariance under small variations $\delta \hat{\boldsymbol{m}}_{i}$. This leads to the condition

$$
\hat{\boldsymbol{m}}_{i} \times\left(\boldsymbol{h}_{i}-S \sum_{j} J_{i j} \hat{\boldsymbol{m}}_{j}\right)=0,
$$

which shows that the magnetization aligns parallel to the sum of external and exchange field, as expected. For given $\boldsymbol{h}_{i}$ and $J_{i j}$, this is a system of non-linear equations for the spin directions $\hat{\boldsymbol{m}}_{i}$ in the classical ground state. To study fluctuations, we expand the transverse components as $\boldsymbol{S}_{i}^{\perp}=\frac{1}{2} \sum_{p= \pm} S_{i}^{-p} \boldsymbol{e}_{i}^{p}$, with spherical basis vectors $\boldsymbol{e}_{i}^{p}=\hat{\boldsymbol{e}}_{i}^{1}+i p \hat{\boldsymbol{e}}_{i}^{2}, p= \pm$, where $\left\{\hat{\boldsymbol{e}}_{i}^{1}, \hat{\boldsymbol{e}}_{i}^{2}, \hat{\boldsymbol{m}}_{i}\right\}$ is

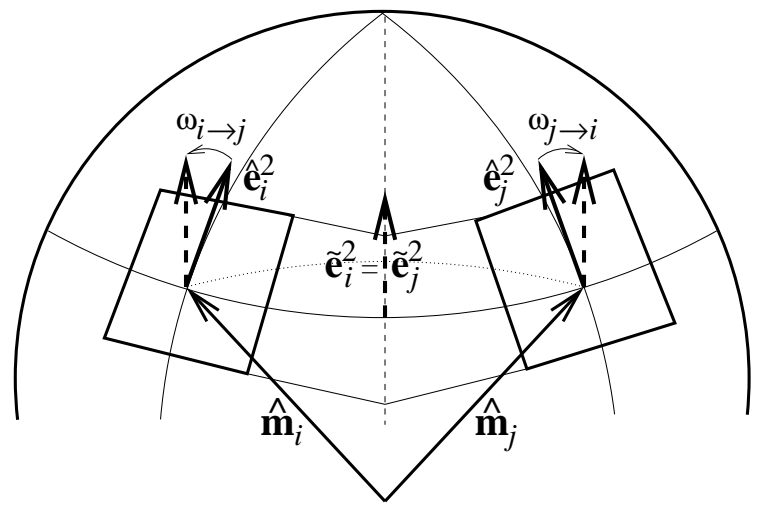

FIG. 2: Definition of the rotated basis vectors and the corresponding rotation angles $\omega_{i \rightarrow j}$ and $\omega_{j \rightarrow i}$. The rotated vectors $\tilde{\boldsymbol{e}}_{i}^{2}=\tilde{\boldsymbol{e}}_{j}^{2}$ lie in the intersection of the two tangent planes associated with $\hat{\boldsymbol{m}}_{i}$ and $\hat{\boldsymbol{m}}_{j}$, shown as a dashed line. The dotted line is the geodesic connecting the two sites on the unit sphere in order-parameter space.

a local orthogonal triad of unit vectors. The transverse part of our spin Hamiltonian can then be written as

$$
\hat{H}^{\perp}=\frac{1}{8} \sum_{i, j} \sum_{p, p^{\prime}} J_{i j}\left(\boldsymbol{e}_{i}^{p} \cdot \boldsymbol{e}_{j}^{p^{\prime}}\right) S_{i}^{-p} S_{j}^{-p^{\prime}}
$$

For explicit calculations, we use the standard representation $S_{i}^{\|}=S-b_{i}^{\dagger} b_{i}$ and $S_{i}^{+}=\left(S_{i}^{-}\right)^{\dagger}=\sqrt{2 S} b_{i}\left[1+O\left(S^{-1}\right)\right]$ in terms of canonical boson operators $b_{i}$. Note that $\hat{H}^{\perp}$ $=O(S)$, whereas $\hat{H}^{\prime}=O\left(S^{1 / 2}\right)$ due to Eq. (7).

While the $\hat{\boldsymbol{m}}_{i}$ are fixed by Eq. (7), there is a remaining local $U(1)$ gauge freedom associated with the rotation of the transverse basis vectors $\boldsymbol{e}_{i}^{p}$. Let us rewrite the scalar product $\boldsymbol{e}_{i}^{p} \cdot \boldsymbol{e}_{j}^{p^{\prime}}$ such that the local gauge invariance is manifest. Rotating the transverse basis vectors with an angle $\omega_{i \rightarrow j}$ around the local normal $\hat{\boldsymbol{m}}_{i}$ leads to $\boldsymbol{e}_{i}^{p}=$ $e^{i p \omega_{i \rightarrow j}} \tilde{\boldsymbol{e}}_{i}^{p}$, as shown in Fig. 2. If we choose the rotated basis vectors $\tilde{\boldsymbol{e}}_{i}^{p}$ and $\tilde{\boldsymbol{e}}_{j}^{p}$ associated with two neighboring points $\hat{\boldsymbol{m}}_{i}$ and $\hat{\boldsymbol{m}}_{j}$ on the unit sphere such that $\tilde{\boldsymbol{e}}_{i}^{2}$ and $\tilde{\boldsymbol{e}}_{j}^{2}$ are equal and parallel to $\hat{\boldsymbol{m}}_{i} \times \hat{\boldsymbol{m}}_{j}$, then $\tilde{\boldsymbol{e}}_{i}^{p} \cdot \tilde{\boldsymbol{e}}_{j}^{p^{\prime}}=$ $\left(\hat{\boldsymbol{m}}_{i} \cdot \hat{\boldsymbol{m}}_{j}-p p^{\prime}\right)$. Rotating back to the original basis, we arrive at

$$
\boldsymbol{e}_{i}^{p} \cdot \boldsymbol{e}_{j}^{p^{\prime}}=\left(\hat{\boldsymbol{m}}_{i} \cdot \hat{\boldsymbol{m}}_{j}-p p^{\prime}\right) \exp \left[i p \omega_{i \rightarrow j}+i p^{\prime} \omega_{j \rightarrow i}\right]
$$

Note that the condition $\tilde{\boldsymbol{e}}_{i}^{2}=\tilde{\boldsymbol{e}}_{j}^{2}$ implies $\operatorname{Im}\left[\tilde{\boldsymbol{e}}_{i}^{p} \cdot \tilde{\boldsymbol{e}}_{j}^{p^{\prime}}\right]=$ 0 . Geometrically, this means that $\tilde{\boldsymbol{e}}_{i}^{p}$ and $\tilde{\boldsymbol{e}}_{j}^{p}$ are related by parallel transport 21] along the shortest path (i.e., a geodesic, corresponding to a rotation around an axis parallel to $\hat{\boldsymbol{m}}_{i} \times \hat{\boldsymbol{m}}_{j}$ ) connecting the points $\hat{\boldsymbol{m}}_{i}$ and $\hat{\boldsymbol{m}}_{j}$ on the surface of the unit sphere. Substituting Eq. (9) into Eq. (8), we obtain

$$
\begin{aligned}
\hat{H}^{\perp} & =\frac{1}{8} \sum_{i, j} J_{i j}\left[\left(1+\hat{\boldsymbol{m}}_{i} \cdot \hat{\boldsymbol{m}}_{j}\right) e^{i\left(\omega_{i \rightarrow j}-\omega_{j \rightarrow i}\right)} S_{i}^{-} S_{j}^{+}\right. \\
& \left.-\left(1-\hat{\boldsymbol{m}}_{i} \cdot \hat{\boldsymbol{m}}_{j}\right) e^{i\left(\omega_{i \rightarrow j}+\omega_{j \rightarrow i}\right)} S_{i}^{-} S_{j}^{-}+\text {h.c. }\right] .
\end{aligned}
$$


This expression is manifestly invariant under the local $U(1)$ gauge transformation $\omega_{i \rightarrow j} \rightarrow \omega_{i \rightarrow j}+\alpha_{i}, S_{i}^{p} \rightarrow$ $S_{i}^{p} e^{i p \alpha_{i}}$, with $\alpha_{i}$ arbitrary. The first term in Eq. (10) shows that a localized spin deviation acquires a phase as it moves between sites, which is due to the site-dependent orientation of the ground-state magnetization. The local gauge invariance implies conservation of the associated current,

$$
\frac{\partial \hat{H}^{\perp}}{\partial \alpha_{i}}=\sum_{j} \frac{\partial \hat{H}^{\perp}}{\partial \omega_{i \rightarrow j}}=-\sum_{j} J_{i j} \hat{\boldsymbol{m}}_{i} \cdot\left(\boldsymbol{S}_{i}^{\perp} \times \boldsymbol{S}_{j}^{\perp}\right) .
$$

To see this explicitly, we use the equation of motion (3) to write

$$
\begin{aligned}
\hbar \frac{\partial S_{i}^{\|}}{\partial t}= & -\sum_{j} J_{i j} \hat{\boldsymbol{m}}_{i} \cdot\left(\boldsymbol{S}_{i}^{\perp} \times \boldsymbol{S}_{j}^{\perp}\right) \\
& -\boldsymbol{S}_{i}^{\perp} \cdot\left[\hat{\boldsymbol{m}}_{i} \times\left(\boldsymbol{h}_{i}-\sum_{j} J_{i j} S_{j}^{\|} \hat{\boldsymbol{m}}_{j}\right)\right]
\end{aligned}
$$

Within linear spin-wave theory, the last term may be neglected since it is an order $S^{-1 / 2}$ smaller than the first term, due to the condition (7). Taking the thermal average of both sides of Eq. (12) and using the fact that equilibrium averages are time-independent, we conclude that the average of Eq. (11) indeed vanishes, corresponding to a longitudinal spin current with vanishing lattice divergence.

However, in a ring geometry there can be a finite circulating spin current in thermal equilibrium provided the classical spin configuration $\boldsymbol{m}_{i}$ covers a finite total solid angle $\Omega$ on the unit sphere in order-parameter space as we move once around the ring. In the case of nearest-neighbor coupling, we obtain the explicitly gauge-invariant expression $\Omega=\sum_{i=1}^{N}\left[\omega_{i \rightarrow i+1}-\omega_{i \rightarrow i-1}\right]$, where $N$ is the number of spins. Note that $\Omega$ can be identified with the total defect angle ("anholonomy") associated with the corresponding parallel transport of a tangential vector along a closed path of geodesics [21].

We now evaluate the spin current for a ferromagnetic Heisenberg chain with nearest-neighbor coupling $J_{i, i \pm 1}=-J<0$. The component of the spin current in the direction $\hat{\boldsymbol{m}}_{i}$, the divergence of which appears in Eq. (12), can be written in the gauge invariant form

$$
I_{s}=-J\left\langle\hat{\boldsymbol{m}}_{i} \cdot\left(\boldsymbol{S}_{i}^{\perp} \times \boldsymbol{S}_{i+1}^{\perp}\right)\right\rangle=-\partial F_{s}(\Omega) / \partial \Omega,
$$

where $F_{s}(\Omega)$ is the free energy of the spin system. On the other hand, the transverse spin current component in the direction of $\hat{\boldsymbol{m}}_{i} \times \hat{\boldsymbol{m}}_{j}$ is an order $1 / N$ smaller than $I_{s}$. In deriving Eq. (13), we have neglected the terms involving the combinations $S_{i}^{-} S_{j}^{-}$and $S_{i}^{+} S_{j}^{+}$in Eq. (10), because for a ferromagnet the contribution of these quantum fluctuations to $I_{s}$ involves higher powers of $1 / N$ which are dominant only for $T \rightarrow 0$. Since the current is a topological property of the system, we may choose any convenient geometry for explicit calculations. For simplicity, we shall assume a radial magnetic field $\boldsymbol{B}_{i}=|\boldsymbol{B}| \boldsymbol{r}_{i} /\left|\boldsymbol{r}_{i}\right|$, with the spins located at constant latitude $\vartheta_{i}=\vartheta$ (see Fig. 11. For $|\boldsymbol{h}| \equiv g \mu_{\mathrm{B}}|\boldsymbol{B}| \gtrsim J S(2 \pi / N)^{2}$, the classical ground-state configuration $\hat{\boldsymbol{m}}_{i}$ is radial as well, with a slightly different latitude $\vartheta_{m}$ satisfying

$$
\sin \left(\vartheta_{m}-\vartheta\right)=-(J S /|\boldsymbol{h}|)[1-\cos (2 \pi / N)] \sin \left(2 \vartheta_{m}\right)
$$

At low temperatures $(T \ll J S)$ we may approximate the magnon energies by $\epsilon_{n}+|\boldsymbol{h}|$ with dispersion $\epsilon_{n}=$ $J S a^{2} k_{n}^{2}$, where $a$ is the lattice spacing and $k_{n}=\frac{2 \pi}{L}(n-$ $\left.\frac{\Omega}{2 \pi}\right)$ are the quantized wavevectors of the magnons on a ring with circumference $L$. To leading order in spin-wave theory, we then obtain the following from Eq. (13) for the magnetization current $I_{m}=\left(g \mu_{\mathrm{B}} / \hbar\right) I_{s}$ :

$$
I_{m}=\frac{g \mu_{\mathrm{B}}}{L} \sum_{n} \frac{v_{n}}{e^{\left(\epsilon_{n}+|\boldsymbol{h}|\right) / T}-1}
$$

where $v_{n}=\hbar^{-1} \partial \epsilon_{n} / \partial k_{n}=2 J S a^{2} k_{n} / \hbar$. Clearly, Eq. (15) is the exact bosonic analogue of Eq. (11) 22].

If the temperature is large compared with the level spacing $\Delta=J S(2 \pi / N)^{2}$ between the ground state and the first magnon excitation, then Eq. (15) can be evaluated analytically,

$$
I_{m}=\frac{g \mu_{\mathrm{B}} T}{\hbar} \frac{\sin \Omega}{\cos \Omega-\cosh (2 \pi \sqrt{|\boldsymbol{h}| / \Delta})}+O\left(e^{-2 \pi \sqrt{\pi T / \Delta}}\right)
$$

which is accurate for $\Delta \ll T \ll J S$. In view of $|\boldsymbol{h}| / \Delta=S\left(g \mu_{\mathrm{B}}|\boldsymbol{B}| / J S^{2}\right)(N / 2 \pi)^{2}$, it is clear that the persistent spin current is a mesoscopic quantum effect, which vanishes for an infinite system or in the classical limit $S \rightarrow \infty$ with constant $J S^{2}$. The radial spin configuration shown in Fig. 11yields $\Omega=2 \pi\left(1-\cos \vartheta_{m}\right)$ to leading order in $1 / N$, so that the factor $|\sin \Omega|$ is maximal for $\cos \vartheta_{m}=3 / 4$ or $1 / 4$, i.e., $\vartheta_{m} \approx 41^{\circ}$ or $76^{\circ}$. For $|\boldsymbol{h}| \approx \Delta$ Eq. (16) predicts a magnetization current on the order of $I_{m} \approx g \mu_{\mathrm{B}} T / \hbar$ around the ring.

Experimentally, the magnetization current can be detected by measuring the electric voltage between two points above and below the ring. The source of a spatially varying magnetization $\boldsymbol{M}(\boldsymbol{r})=\sum_{i} \delta\left(\boldsymbol{r}-\boldsymbol{r}_{i}\right) \boldsymbol{m}_{i}$ is an effective current density $\boldsymbol{j}(\boldsymbol{r})=c \nabla \times \boldsymbol{M}(\boldsymbol{r})$. If the magnetization is moving with velocity $\boldsymbol{v}(\boldsymbol{r})$, it is accompanied by a polarization $\boldsymbol{P}(\boldsymbol{r})=\frac{\boldsymbol{v}(\boldsymbol{r})}{c} \times \boldsymbol{M}(\boldsymbol{r})$ to leading order in $\boldsymbol{v}(\boldsymbol{r}) / c$. This is easily shown by means of a Lorentz boost to the rest frame of the magnetic dipoles 23]. The polarization corresponds to a charge density $\rho(\boldsymbol{r})=-\nabla \cdot \boldsymbol{P}(\boldsymbol{r})$, which in turn generates an electric field $\boldsymbol{E}(\boldsymbol{r})=-\nabla \phi(\boldsymbol{r})$, with the scalar potential $\phi(\boldsymbol{r})=\int d^{3} r^{\prime} \rho\left(\boldsymbol{r}^{\prime}\right) /\left|\boldsymbol{r}-\boldsymbol{r}^{\prime}\right|$. Combining the above relations, we obtain a generalized Biot-Savart law for the 


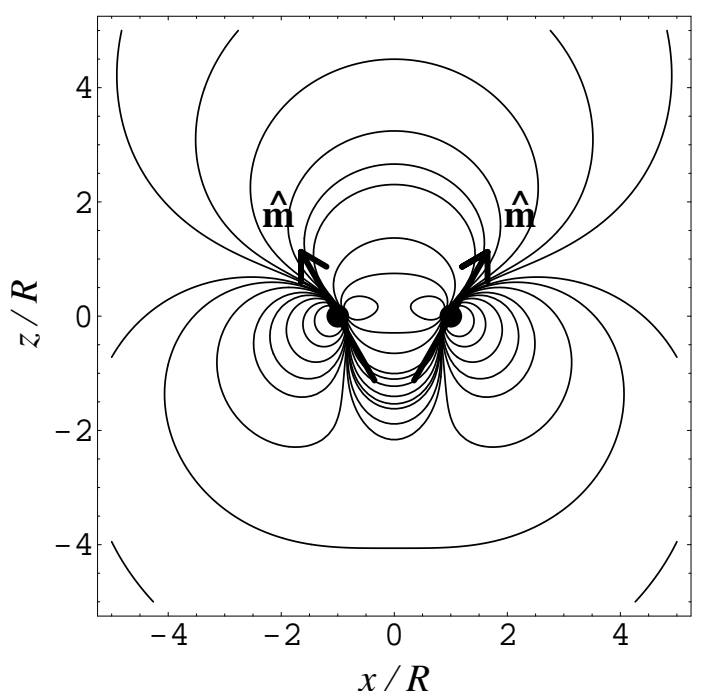

FIG. 3: Lines of constant electric potential due to a spin current circulating in a mesoscopic Heisenberg ring with radius $R$ in the geometry of Fig. 1 for $\vartheta_{m}=30^{\circ}$.

scalar potential due to magnetic dipole currents,

$$
\begin{aligned}
\phi(\boldsymbol{r}) & =\frac{1}{c} \int d^{3} r^{\prime}\left[\boldsymbol{v}\left(\boldsymbol{r}^{\prime}\right) \times \boldsymbol{M}\left(\boldsymbol{r}^{\prime}\right)\right] \cdot \frac{\left(\boldsymbol{r}-\boldsymbol{r}^{\prime}\right)}{\left|\boldsymbol{r}-\boldsymbol{r}^{\prime}\right|^{3}} \\
& =\frac{I_{m}}{c} \oint\left[d \boldsymbol{r}^{\prime} \times \hat{\boldsymbol{m}}\left(\boldsymbol{r}^{\prime}\right)\right] \cdot \frac{\boldsymbol{r}-\boldsymbol{r}^{\prime}}{\left|\boldsymbol{r}-\boldsymbol{r}^{\prime}\right|^{3}} .
\end{aligned}
$$

The second line is valid for a current loop, where $\boldsymbol{v}|\boldsymbol{M}| d^{3} r=I_{m} d \boldsymbol{r}$. Although Eq. (17) is of fundamental importance in spin transport, we have not been able to find it in standard texts on classical electrodynamics. The expression derived in Ref. 19 for a straight line is a special case of Eq. (17). For the simple geometry shown in Fig. 1 the integration in Eq. (17) can be reduced to elliptic integrals. The equipotentials are shown in Fig. [3] In the far zone, the electric field approaches a dipole field, with potential $\phi(\boldsymbol{r})=\boldsymbol{p} \cdot \boldsymbol{r} /|\boldsymbol{r}|^{3}$ and dipole moment $\boldsymbol{p}=-\boldsymbol{e}_{z}\left(I_{m} / c\right) L \sin \vartheta_{m}$. To estimate the order of magnitude of the magnetization current, consider a mesoscopic $S=1 / 2$ Heisenberg chain with $g=2, N=100$, and $J=100 \mathrm{~K}$. Then the condition $g \mu_{\mathrm{B}}|\boldsymbol{B}| \approx \Delta$ is satisfied for $|\boldsymbol{B}| \approx 0.1 \mathrm{~T}$. To obtain a sizable $\Omega$, one should generate inhomogeneities of the magnetic field in the submicron range; these may be achievable in the vicinity of a magnetic flux line trapped in a type-II superconductor. In the dipole approximation, the potential drop between two points located a distance $d$ above and below the loop on the $z$-axis is given by $U \approx 0.5 \mathrm{nV} \cdot(T / \mathrm{K})(L / \mathrm{nm}) /(d / \mathrm{nm})^{2}$. For $T=60 \mathrm{~K}$ and $d=L=100 \mathrm{~nm}$ this yields a voltage $U \approx 0.3 \mathrm{nV}$. However, its experimental detection is difficult, because mobile charges will tend to screen this static dipole field. Note that the magnetization current in Eq. (15) involves only a single factor of $g \mu_{\mathrm{B}}$, whereas for a two-terminal geometry the current is proportional to $\left(g \mu_{\mathrm{B}}\right)^{2}$ [19].
In summary, we have shown that a ferromagnetic Heisenberg ring in an inhomogeneous magnetic field can support a persistent magnetization current, which is the precise bosonic analogue of the persistent charge current in normal metal rings. The magnetization current is a mesoscopic quantum interference effect and flows without dissipation, corresponding to an infinite spin conductance. For weak magnetic fields $\left(g \mu_{\mathrm{B}}|\boldsymbol{B}| \approx \Delta\right)$ the magnetization current gives rise to an electric dipole field. For larger fields, the ballistic contribution to the spin current considered in this work is exponentially suppressed. By analogy with the persistent charge current in normal metal rings, we expect that in this regime the persistent magnetization current is dominated by collective phenomena such as spin diffusion and weak localization effects. The calculation of the dominant contribution in this regime is still an open problem. With suitable magnetic fields, persistent magnetization currents should also exist in antiferromagnetic or ferrimagnetic spin chains, where we expect finite currents at $T=0$ due to quantum fluctuations.

This work was supported by the DFG via Forschergruppe FOR 412, Project No. KO 1442/5-1.

[1] Y. Imry, Introduction to Mesoscopic Physics (Oxford University Press, Oxford, 1997).

[2] F. Hund, Ann. Phys. (Leipzig) 32, 102 (1938).

[3] M. Büttiker, Y. Imry, and R. Landauer, Phys. Lett. 96A, 365 (1983).

[4] L. P. Lévy, G. Dolan, J. Dunsmuir, and H. Bouchiat, Phys. Rev. Lett. 64, 2074 (1990).

[5] V. Chandrasekhar, R. A. Webb, M. J. Brady, M. B. Ketchen, W. J. Gallagher, and A. Kleinsasser, Phys. Rev. Lett. 67, 3578 (1991).

[6] D. Mailly, C. Chapelier, and A. Benoit, Phys. Rev. Lett. 70, 2020 (1993).

[7] H.-F. Cheung, Y. Gefen, and E. K. Riedel, IBM J. Res. Develop. 32, 359 (1988).

[8] Z. Cao, X. Yu, and R. Han, Phys. Rev. B 56, 5077 (1997).

[9] Y. Aharonov and A. Casher, Phys. Rev. Lett. 53, 319 (1984).

[10] See, for example, D. D. Awschalom, D. Loss, and N. Samarth (Eds.), Semiconductor Spintronics and Quantum Computation, (Springer, Berlin, 2002).

[11] D. Loss, P. Goldbart, and A. V. Balatsky, Phys. Rev. Lett. 65, 1655 (1990).

[12] A. Stern, Phys. Rev. Lett. 68, 1022 (1992).

[13] X.-C. Gao and T.-Z. Qian, Phys. Rev. B 47, 7128 (1993).

[14] J. König, M. C. Bonsager, and A. H. MacDonald, Phys. Rev. Lett. 87, 187202 (2001).

[15] G. Tatara and H. Kohno, Phys. Rev. B 67, 113316 (2003).

[16] A. G. Mal'shukov, C. S. Tang, C. S. Chu, and K. A. Chao, cond-mat/0211559

[17] S.-Q. Shen and X. C. Xie, Phys. Rev. B 67, 144423 (2003).

[18] L. Y. Gorelik, R. I. Shekhter, V. Vinokur, D. Feldman, 
V. Kozub, and M. Jonson, cond-mat/0211563

[19] F. Meier and D. Loss, Phys. Rev. Lett. 90, 167204 (2003).

[20] Note that the spin current is a second rank tensor. We call $\hat{\mathbf{m}}_{i} \cdot \mathbf{I}_{i \rightarrow j}$ the longitudinal spin current, where longitudinal refers to spin space.

[21] See, for example, A. Shapere and F. Wilczek, Geometric Phases in Physics, (World Scientific, Singapore, 1989).
[22] To compare Eqs. (1) and (15), we should divide the currents by the corresponding "charges", i.e., the particle number current $I /(-e)$ should be compared with $I_{s} / \hbar=I_{m} /\left(g \mu_{\mathrm{B}}\right)$.

[23] J. E. Hirsch, Phys. Rev. B 60, 14787 (1999). 